\title{
Design of a Novel Multifunction Decision Support Display for Anesthesia Care: AlertWatch ${ }^{\circledast}$ OR
}

Kevin K. Tremper ${ }^{1 *}$ D, Jenny J. Mace ${ }^{1}$, Jan M. Gombert ${ }^{1}$, Theodore T. Tremper ${ }^{1}$, Justin F. Adams ${ }^{2}$ and James P. Bagian'

\begin{abstract}
Background: This paper describes the design of a multifunction alerting display for intraoperative anesthetic care. The design was inspired by the multifunction primary flight display used in modern aviation.

Results: The display retrieves live data from multiple sources; the physiologic monitors, the anesthesia information management system, the laboratory values and comorbidities from patient's problem summary list, medical history or history \& physical. This information is integrated into a display composed of readily identifiable icons of organ systems, which are color coded to signify normal range, marginal range, abnormal range (by green, yellow, red respectively) and orange outlines for comorbidities/risk factors. There are dozens of text alerts, which can be presented as black text (informational), red text (important information) and red scrolling text (highest importance information). The alerts are derived from current standards in the literature and some involve complex calculations being conducted in the background.
\end{abstract}

Conclusions: The goal of such a system is to improve the quality and safety of anesthetic care by providing enhanced situational awareness in a fashion analogous to the "glass cockpit" and its primary flight display which has improved aviation safety.

Keywords: Decision support, Integrated medical display, Anesthesia alerting system

\section{Background}

The practice of anesthesia is often described in terms similar to that of being a pilot; the preop evaluation is the flight plan, the induction is the take-off, the performance of the procedure is cruise and the emergence is the landing. During each of these phases and during the "flight" the pilot/ anesthesiologist follows the flight path of the procedure by relying on an array of instruments providing live data to assess the stability of the aircraft/patient. The aviation industry has led all industries in the area of safety and quality [1]. They have also led in the design of the cockpit from an increasing array of analog dials presenting data from an increasing number of sensors, to a more functional and intuitive display known as the multifunctional display or "glass cockpit [2]." This glass cockpit consolidates

\footnotetext{
* Correspondence: ktremper@umich.edu

${ }^{1}$ Department of Anesthesiology, University of Michigan Medicine, $1500 \mathrm{E}$.

Medical Center Drive, Ann Arbor, MI 48109, USA

Full list of author information is available at the end of the article
}

information from hundreds of sensors to three displays and has been associated with a significant improvement in aviation safety [1]. The three displays are the Navigation, the System's Operations (displaying hydraulic pressures and other systems issues on the aircraft), and in front of the pilot and co-pilot, the multifunction display known as the Primary Flight Display. This integrated display provides an increased level of situational awareness by showing an integrated representation of the horizon, the ground, sky, which changes attitude showing pitch and roll of the aircraft, and a limited but important number of digital data such as airspeed and altitude. Figure 1 In the background multiple inputs of data are analyzed and presented when needed, in the order of importance. This system has enabled pilots to fly the plane and simultaneously address issues, which allows them to maintain "situational awareness" even during unplanned emergency situations. Medicine, and Anesthesiology in particular, has again followed the aviation industry with 


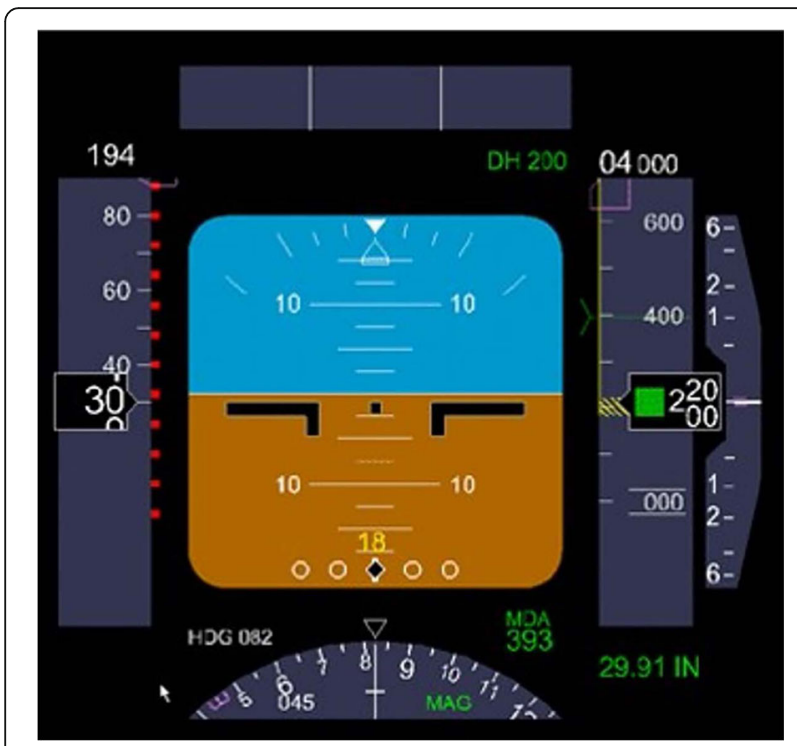

Fig. 1 The Multifunction Monitor. In the 1980s aviation switched from multiple analog gauges to the "glass cockpit" with a multifunction monitor as the primary flight display. Above is the primary fight flight display of the Boeing 737 aircraft. It presents the horizon (blue sky/brown earth) to provide a visual assessment of yawl and pitch. There are also algorithms analyzing data which it presents when needed in the hierarchy of importance. [Primary Flight Display. (Accessed on August 9, 2016, at https://en.wikipedia.org/wiki/ Primary_flight_display)]

advanced monitoring standards, emergency protocols, team training, simulators and checklists; not only in the operating room but in all procedural areas [3-5].

With the broad implementation of the electronic medical record systems, which for anesthesiologists is known as the Anesthesia Information Management System (AIMS), anesthesiologists may now manage live digital data provided continuously and permanently stored. The proliferation of AIMS and other structured electronic medical record (EMR) systems, which include patient co-morbidities and outcomes in addition to intraoperative data, has resulted in a proliferation of observational studies finding associations between types of intraoperative care and postoperative outcomes [6-13]. The results of these studies may be applied in real-time during anesthesia care, but only if the information is made available to the provider at the time it is appropriate. That is, algorithms detecting labs, co-morbidities and physiologic data, which fall out of agreed upon standards can automatically trigger alerts in real time. As a result, live analysis can provide alerts to improve care in real time when there is the opportunity to improve the care of the patient, where reoccurring retrospective quality assessments may fall short. Examples including, but not limited to, glucose, blood pressure, ventilation and overall fluid management which can be assisted by live calculations and live alerts [6-13].

In an attempt to imitate what has been found successful in the aviation industry a multifunction display has been developed for operating room anesthesia [14-16]. AlertWatch ${ }^{\circ} \mathrm{OR}$ is a multifunction display, driven by live data extracted from physiologic monitors and the EMR, displayed in a readily identifiable icon view of organ systems, Fig. 2 [14, 15]. This display extracts, analyzes and presents over 250 pieces of information, providing a "live" organ system view with a beating heart, expanding/contracting aortic arch and ventilating lungs. The display is color coded to indicate normal range (green), marginal range (yellow), and abnormal range (red) for the data related to each organ system or lab value. Organs or systems with co-morbidities are outlined in orange. The display also has dozens of digital text alerts and two audible alerts (e.g. low or missing blood pressure). The text alerts which appear in the upper right alert section of the display come in three hierarchies: black text for basic information, red text for important information, and scrolling red text for information that should be addressed immediately. There are multiple calculations being conducted in the background for clinical purposes [17], AlertWatch is a Food and Drug Administration (FDA) cleared decision support software device [17]. Because of the high incidence of colorblindness and as part of the FDA clearance process, the application has been tested using software that mimics colorblind individuals [18]. In addition, to ensure the orange co-morbidities are not confused with other color changes within organs and labs, the orange colors are highlighted around the organ or the lab, not filling the space thereby differentiating by the location. Most values and alerts are presented, not only by color changes, but digital changes and as stated above by scrolling text alerts and some audible alerts. The purpose of this current report is to present the design specifications and rational for display elements, algorithms, calculations and alerts in AlertWatch ${ }^{\circ}$ OR version 2.60. As software development is an iterative process, elements of the AlertWatch system as described in this report are subject to change in future updates. A demo of the system can be viewed on the website: http://www.alertwatch.com/

\section{Implementation}

The primary purpose of AlertWatch is to extract data from multiple sources and display these inputs and other analyzed information in a readily interpretable manner so that clinicians can be made aware of the ever changing status of any patient in real-time. These live data extracts come from multiple sources within the hospital systems including: the admission \& discharge system, physiologic data, the laboratory system, AIMS event documentation, as well as co-morbidities from the anesthesia history \& physical or diagnosis codes from the patient's problem list.

The physiologic data may be captured in a number of ways depending on institutional preference. Data retrieved from AIMS are updated less frequently than data captured 


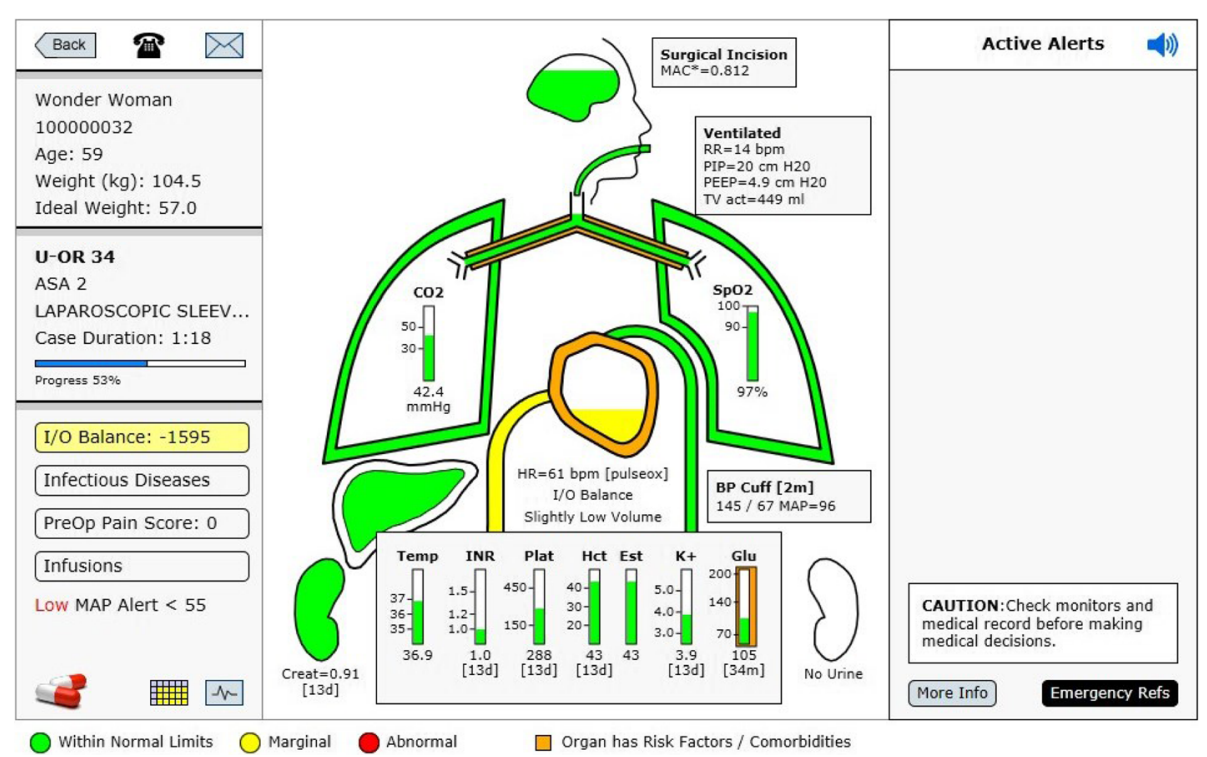

Fig. 2 The AlertWatch ${ }^{\oplus}$ Display. The above AlertWatch ${ }^{\circledR}$ display is analogous to the primary flight display in aviation, Fig. 1. It receives information from physiologic monitors, AIMS, patients' history and physical for co-morbidities and laboratory systems and displays this information in readily identifiable icons of human organs. It is color coded to signify: normal range (green), marginal (yellow), and abnormal range (red). Organs highlighted in orange have comorbidities. The left-side of the display provides patient case data, calculated intake and output (I\&O) balance, presence of infectious disease, preoperative pain score and the presence of infusions. Text alerts are presented on right-side of the display in the following hierarchy: black text are informational, red text for important alerts and scrolling red text for those which need immediate attention, this figure has no text alerts, see Fig. 4a. There are 54 alerts originating from the 250 pieces of information continuously being extracted from the medical records. No data are stored in AlertWatch ${ }^{\oplus}$, just retrieved from the EMR and presented. The central portion of the display is composed of icons of organ systems: brain, lungs, heart, liver kidneys. The color of the brain and level within the brain signifies the age-adjusted anesthetic level while the level within the heart signifies the fluid status with respect to I\&O balance; green $+/-20 \%$ of estimated blood volume (EBV), yellow $+/-40 \%$ of EBV (high or low level) and red $\geq 40 \%$ EBV positive or negative (very high or very low level). This patient has an $1 \& 0$ of -1595 so is in the yellow range and the heart is filled to the low yellow level. If there is an invasive arterial catheter or central venous catheter the fluid level and color within the heart will be guided by the normal ranges of SPV or CVP. The Green, Yellow and Red ranges for all variables are configurable. This patient has lung and cardiac disease signified by the orange outline. They also have diabetes noted by the orange outline of the glucose. All ranges/colors are presented in Additional File 1. For a more complete description go to the website go to the website: http://www.alertwatch.com

directly from physiologic systems. The AIMS events and laboratory data are updated minute-by-minute and extracted from the AIMS and laboratory systems. The history \& physical data, including coexisting diseases and airway exam, are extracted from the anesthesia history \& physical or in some cases from standardized codes, such as international classification of diseases (ICD-10) or SNOMED. These comorbidities are categorized by organ system for display on the patient view, Fig. 2. Multiple types of data are extracted from the AIMS and utilized for calculations and display. These include fluid management information, anesthetic administration, ventilator data, etc. Each of these data extracts will be discussed in detail in sections below. AlertWatch ${ }^{\circ}$ is a web-based decision support display, configurable to work with most AIMS and EMR systems.

\section{Results and design Census view}

AlertWatch ${ }^{\oplus}$ opens to a view of the OR census. This is a surveillance view that displays all the operating rooms being monitored, Fig. 3. Each rectangle represents an operating room (OR). If there are multiple sets of operating rooms they can be viewed by clicking on the upper left of the panel noting a drop-down list for all OR locations, Fig. 3. If a provider is logged into any cases at that time their census will only display their cases.

Each operating room rectangle provides information regarding OR number/location, the anesthesia providers in that OR and a track bar noting the case duration, Fig. 3. The track bar moves from left to right as the case proceeds and changes color depending on whether the case is on time (green) delayed (yellow) or very delayed (red), Fig. 3. The background color of the rectangle notes the status of the case: gray (no cases in the room), blue (anesthesia start), $\tan$ (induction end and surgery underway) and peach (post anesthesia care unit [PACU] called for). When the patient leaves the OR the number of minutes the OR has been empty is displayed, Fig. 3.

A series of icons are displayed in the lowest segment of the OR rectangle, Fig. 3. If there is an informational alert, a black square is displayed if there is a more urgent alert the square is red. A purple dot indicates that there 


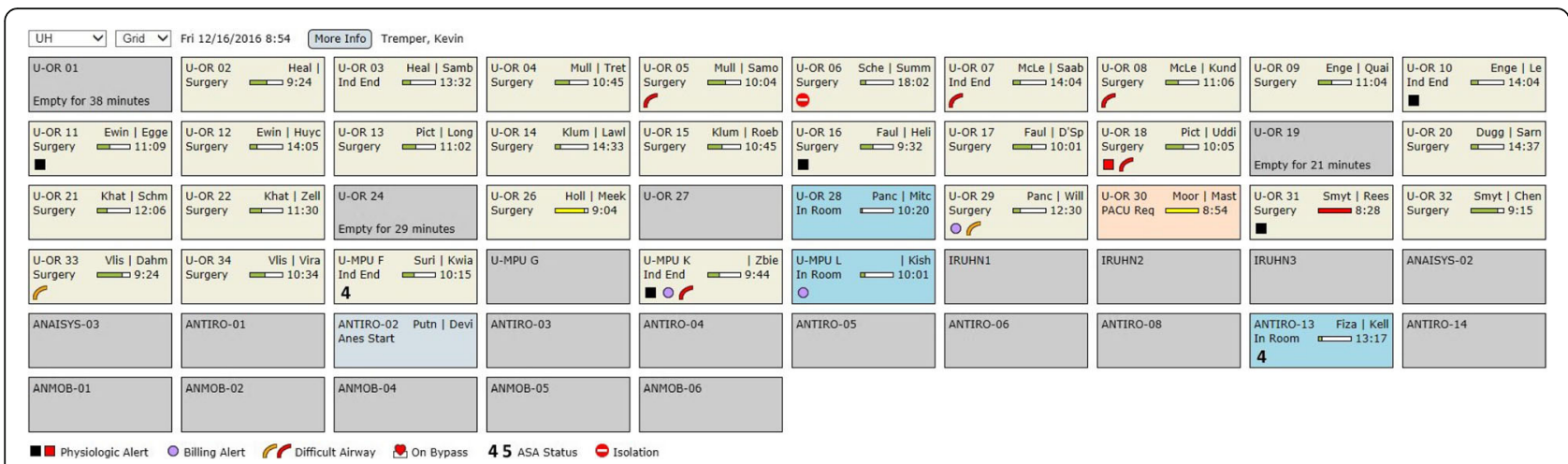

Fig. 3 AlertWatch ${ }^{\circledast}$ Surveillance View. Above is the Surveillance/OR Census View of AlertWatch ${ }^{\circledast}$; each square is an individual operating room. The anesthesia providers are in the upper left corner and the progress of the case is indicated by the colored scroll bar: green if on time, yellow if delayed, and red if delayed more than an hour. The background color of the square designates whether the patient is in the room/anesthesia start (blue), induction complete, surgery underway (tan), PACU called for (peach), or OR empty (gray). When the patient leaves the OR the room is gray and the number of minutes the OR has been empty is displayed. At the base of the OR rectangle icons indicate if there are text alerts (black or red), missing documentation, airway history, on cardiopulmonary bypass, ASA 4 or 5 and Isolation Precautions

is missing documentation. An orange elbow (representing the airway) represents risk factors for a difficult airway and a red elbow represents history of a difficult airway. The heart icon signifies the patient is on cardiopulmonary bypass. A number 4 or 5 represents the patient's American Society of Anesthesiologists (ASA) status. Finally, the red circle icon with a white line through it represents isolation precautions. At the top left of center of the display there is a "More Info" button that links to a full user's manual.

\section{Patient view}

If you click on any OR icon an individual patient view from that OR is displayed. It has three distinct sections, the left side of the screen shows patient information, the right side of the screen shows active alerts, in the center is the live icon view of the patient's organ system display "the primary flight display", Fig. 2. Data are from real patients but all names and registration numbers are fictitious so the patients cannot be identified from these data. Additionally, no date of surgery is supplied.

The left section is the patient information, showing the patient's name and registration number, age, weight and the calculated ideal body weight (calculated based on height and gender) [19]. In the upper left above the patient's name is an icon of a telephone. It opens a window showing the anesthesia providers in the room with their pager numbers/ phone numbers and the telephone number of the OR/ Anesthesia machine, Fig. 2. To the right of the telephone is an envelope icon for sending messages to the AlertWatch ${ }^{\circ}$ team for questions or suggestions.

In the section below the patient's information is the OR number, the ASA status and the surgical procedure. Below the procedure is the case duration in hours and below that is a horizontal scroll bar designating the duration of the case relative to the schedule duration. Below the duration line is \% of the case completed relative to the scheduled case time, Figs. 2 and 3.

Continuing down the far left side of the display is the I \&O Balance, Infectious Disease, Preoperative Pain Score and Infusions, Fig. 2.

If the calculated I\&O balance is within $20 \%$ of the calculated estimated blood volume (EBV), the background color of that button is green [20]. If the calculated fluid balance is between $+/-20 \%$ to $+/-40 \%$ of EBV the background is yellow. If the calculated $1 \& \mathrm{O}$ balance is greater than $40 \%$ positive or negative relative to the EBV the background is red, Fig. 4a (also see Fig. $7 \mathrm{a}-\mathrm{c}$ ).

When you click the I\&O button the detailed calculation is presented, Fig. 4b. The fluid losses (outputs) are: insensible loss, blood loss, third space surgical loss and urine output. The inputs are: crystalloids, colloids, blood and blood products. All ins and outs are based on crystalloid equivalents. Fluid administration with albumin, blood administration and loss are calculated in the following ratios relative to crystalloid, 1.5:1 and 3:1 respectively [21]. The insensible losses are estimated using the 4-2-1 rule. The 4-2-1 rule refers to the estimation of water losses due to respiration. The most common way of estimating this loss is to assume $4 \mathrm{ml} / \mathrm{kg} / \mathrm{h}$ for the first $10 \mathrm{k}, 2 \mathrm{ml} / \mathrm{kg} / \mathrm{h}$ for the next $10 \mathrm{k}$ and $1 \mathrm{ml} / \mathrm{kg} / \mathrm{h}$ for the remaining kilos of body weight. The hourly water loss is multiplied by the NPO time. (22) The insensible loss defaults to $8 \mathrm{~h}$ of nothing by mouth (NPO). The actual hours of NPO can be selected from a drop down list, Fig. 4b. The surgical third space loss defaults to zero, but a drop-down list allows for a selection of three levels of loss; low $(3 \mathrm{ml} / \mathrm{kg} / \mathrm{h})$, medium $(5 \mathrm{ml} / \mathrm{kg} / \mathrm{h})$ and high $(7 \mathrm{ml} / \mathrm{kg} / \mathrm{h})$, Fig. $4 \mathrm{c}$ [22].

The calculation assumes no intravenous fluid has been given prior to the start of the procedure and therefore 
a

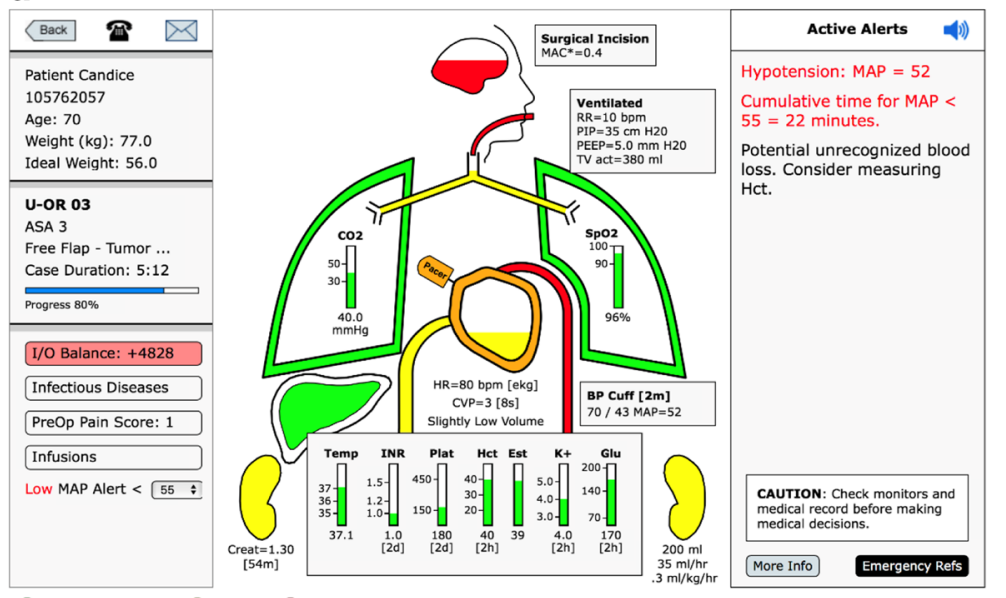

W Within Normal Limits O Marginal Abnormal $\square$ Organ has Risk Factors / Comorbidities

Click the various organ systems, buttons, and bar charts for more information. Not all features are enabled.

b

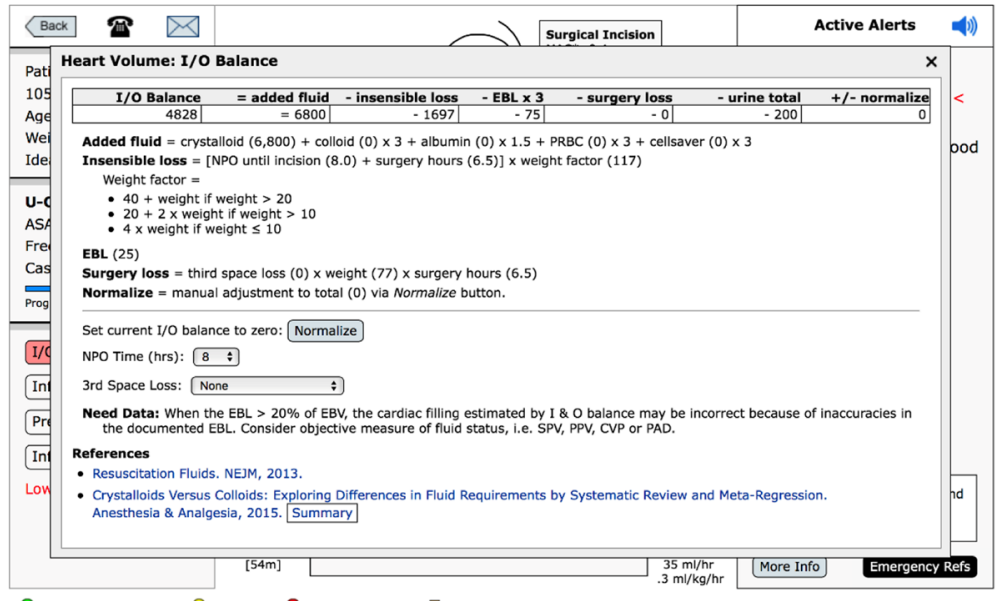

O Within Normal Limits O Marginal - abnormal $\square$ Organ has Risk Factors / Comorbidities

Click the various organ systems, buttons, and bar charts for more information. Not all features are enabled.

C

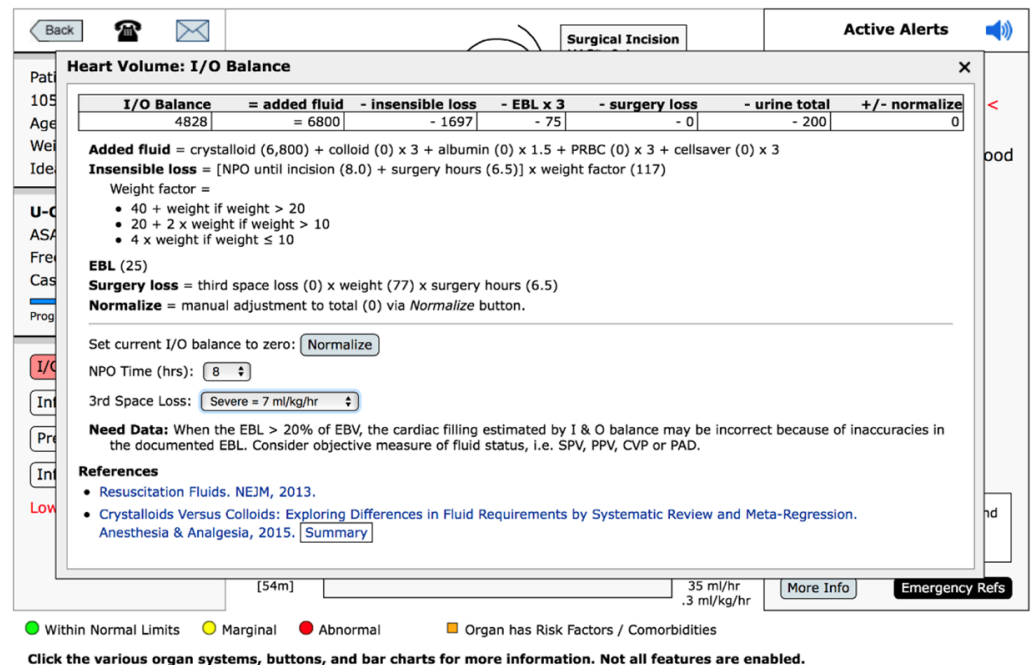

Fig. 4 (See legend on next page.) 


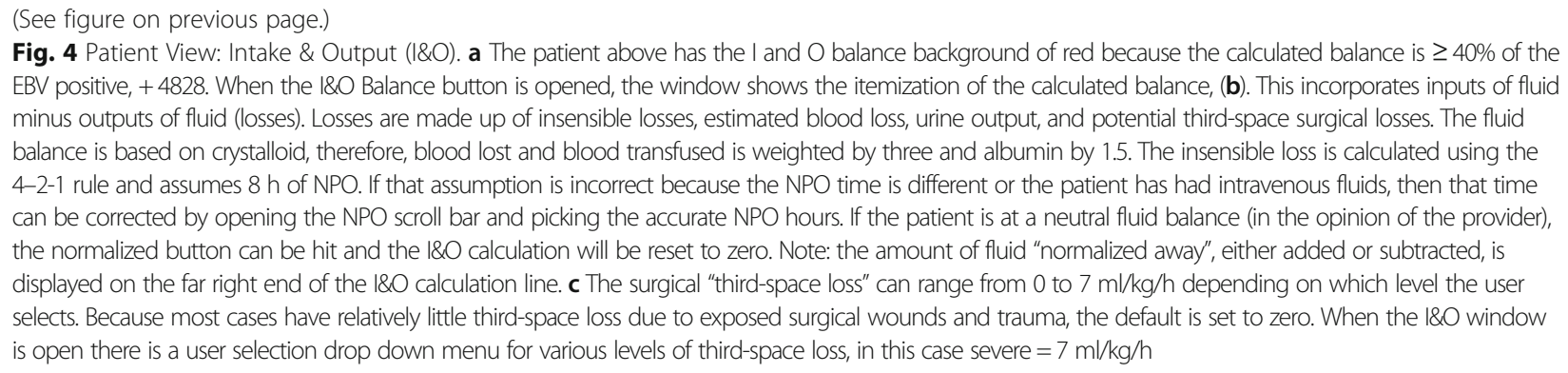

maybe inaccurate, i.e. too negative. For that reason, (or any reason the clinical provider thinks the current $I \& O$ balance is zero) there is a "normalize button" which will "zero out" all the I\&O calculations. If the normalize button has been activated the amount of fluids either positive or negative that has been "normalized away" is displayed on the far right of the I\&O balance at the top. All windows within AlertWatch ${ }^{\circ}$ have the links to the pertinent literature references at the bottom of the window. If there are no invasive measures of intravascular volume resuscitation being employed (e.g. SPV, CVP) then the heart icon will use the calculated I\&O balance to determine the fluid level inside the heart icon, see next section, Fig. 2.

Below the I\&O button is the Infectious Disease button. If the patient has an infectious disease or contact precaution that button will be highlighted in orange, and a red circle icon will be displayed on the census OR square. Selecting the Infectious Disease button displays the specific disease.

The next button is the PreOp Pain Score. If the score is $\geq 4$, the button will be yellow; and if the patient has buprenorphine on their home medications list, the button will be orange.

The lowest button in this section identifies Infusions. If the patient is receiving an anesthetic infusion the button is colored green, if the patient is receiving a vasopressor or insulin infusion the button is yellow, and if the patient is receiving an epinephrine or high dose vasopressor infusions the button is red. Hitting this button will show the infusions.

At the bottom mean arterial pressure (MAP) low alert value is displayed. This can be changed by the provider on a case-by-case basis.

\section{Body icon view}

The central portion of the display shows icons of the major organ systems, labs and the endotracheal tube. If an organ system or lab is colored / highlighted in orange that signifies a comorbidity associated with that organ system or lab, Fig. 2.

At the very bottom of the display the keys for green, yellow, and red; if that key is selected all the ranges will be displayed. These ranges are configurable on installation at an institutional level not for individual clinical providers, Additional File 1.

\section{Brain}

The outline at the top of the figure is the brain icon. If the brain is highlighted in orange it designates there is history of a stroke or risk factors for stroke. Clicking the brain will open up a window showing the detail. Also in the window is a link to the reference used to determine the displayed minimum alveolar concentration (MAC*) value, discussed below. There are also links to emergency protocols.

The "color level" within the brain is the calculated $\mathrm{MAC}^{*}$ value, if the MAC* is equal to or greater than 1.0 the brain will be green and full, Fig. 5. As the MAC* decreases, the green level lowers until the MAC* is $<0.6$. At this point the level turns yellow and when $M A C^{*}$ reduces to $<0.4$ the level turns red, Fig. $4 \mathrm{a}$. MAC* is a calculated anesthetic level associated with postoperative recall, i.e. MAC represents a risk for recall not the traditional MAC calculation for inhaled anesthetics [23]. This cumulative MAC value for inhaled agents is age adjusted [23]. The equation for $\mathrm{MAC}^{*}$ was used in a postoperative recall study conducted in three centers in over 22,000 patients [24]. Mashour, et al. determined the calculated $\mathrm{MAC}^{*}$ value which was associated with an increasing incidence of patient recall. This calculated $\mathrm{MAC}^{*}$ value incorporates both inhaled agents and intravenous agents. The inhaled anesthetic agents expired data are retrieved from the infrared analyzer on the anesthesia machine. The intravenous agents are received from the AIMS documentation of propofol and /or dexmedetomidine infusions. In addition, when an infusion anesthetic (propofol) is used in the MAC calculation it is stated in the MAC* box to the right of the brain, Fig. 5 . There could be an error in over calculating the MAC if the infusion anesthetic were documented but discontinued, (pump problem, intravenous (IV) disconnect, etc.). Because this situation would be most severe if a patient was relaxed with a non-depolarizing muscle relaxant and had no inhaled agent, there is an alert on the display: "Pure TIVA documented. Neuromuscular blockade 


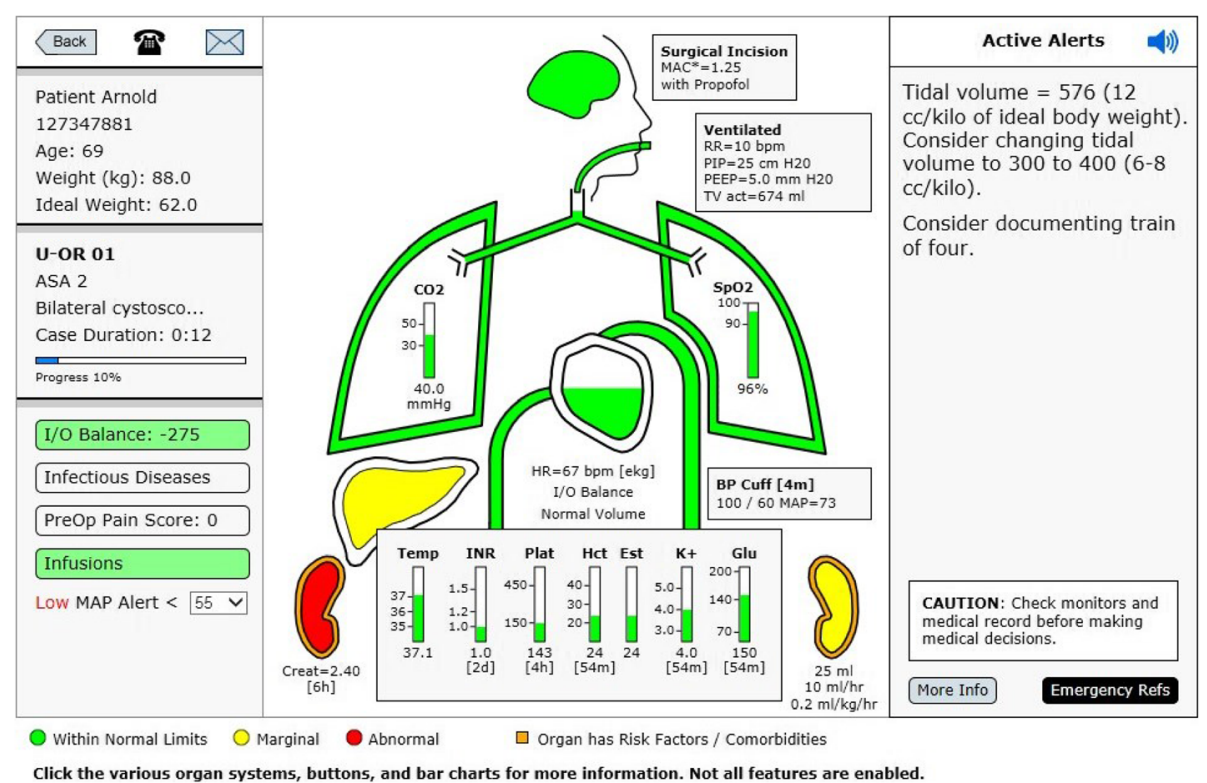

Fig. 5 Brain Minimum Alveolar Concentration (MAC*) Level. The "fluid level" in the brain represents the level of anesthetic. When the calculated, age-adjusted MAC* level is $\geq 1.0$ the brain color is green and full, Fig. 5. The level will lower until the MAC* level reaches 0.7 , at which point the brain color will turn yellow, and as the level moves lower the brain will turn red when the MAC* is $<0.4$, as in Fig. 4a. The $M A C^{*}$ is not the traditional MAC* associated with purely inhalation agents. It is the MAC* that has been associated with the incidence of postoperative recall and includes not only the additive value of inhaled anesthetic agents, but also intravenous agents such as propofol and dexmedetomidine $[23,24]$

given. Consider bispectral index (BIS) or nitrous oxide", Additional File 2, Category:Drugs. In addition, the large prospective trial referred to above excluded all patients under pure TIVA [24].

\section{Endotracheal tube (ETT)}

The endotracheal tube (ETT) icon below the brain will appear clear in color if there is no airway assessment and no history of intubation, green if there is a history of easy mask (grade one or two) and easy intubation (grade one or two view) [25]. If there are risk factors for potential difficulty with airway management, including mask or difficult intubation, the endotracheal tube is orange. If there is a history of a difficult airway: (grade 3 or 4 mask or grade 3 or 4 view, use of an intubating stylet, use of video laryngoscopy or awake intubation) the ETT is red, Fig. 6a [26]. If you open the window of the ETT, the specific airway history and risk factors are displayed, Fig. 6b. There are also emergency references for unanticipated difficult airway in adults and pediatric patients along with literature references $[27,28]$.

\section{Ventilator data}

If the patient is being ventilated with positive pressure ventilation, a box will appear to the right of the ETT showing the ventilation data: respiratory rate, peak inspiratory pressure (PIP), positive end expiratory pressure (PEEP), and tidal volume (TV), Fig. 5.

\section{Lungs}

Below the ETT is the trachea and right and left main bronchia, Fig. 5 . The lungs inflate and deflate with the respiratory rate. The color within the bronchus signifies PIP. If PIP is $\geq 35 \mathrm{~cm} \mathrm{H} 2 \mathrm{O}$, it is yellow and $\geq 40 \mathrm{mmHg}$, it is red. The outline of the Lungs represents the PEEP, green with PEEP 0 to 10 (in adults there is a text alert if PEEP is $<4 \mathrm{~cm} \mathrm{H2O}$ ), yellow with PEEP $\geq 10$ and $<15$, and red when PEEP is $\geq 15 \mathrm{~cm} \mathrm{H} 2 \mathrm{O}$. In the right lung is the saturation value $(\mathrm{SpO} 2)$, and in the left lung is the end-tidal $\mathrm{CO} 2$ (ETCO2) value, Fig. 2.

If the trachea/bronchi are orange, the patient has a history of pulmonary disease. Clicking the trachea/bronchi highlights the pulmonary risk factors and pulmonary diseases e.g. asthma, chronic obstructive pulmonary disease (COPD), smoking history. It also has emergency protocols for bronchospasm and hypoxia as well as emergency pulmonary protocols for adults and children. If the TV is $\geq 10 \mathrm{ml} / \mathrm{kg}$ ideal body weight an alert will be displayed on the right with the recommended TV based on recent literature, Fig. 5 [8].

\section{Heart}

The heart beats/contracts with the heart rate which is either driven from the electrocardiogram (EKG), if available, or the $\mathrm{SpO} 2$ pulse rate if the EKG is not available, Fig. $7 \mathrm{a}-\mathrm{c}$. If there is an orange outline on the heart it signifies comorbidity. Clicking on the heart 


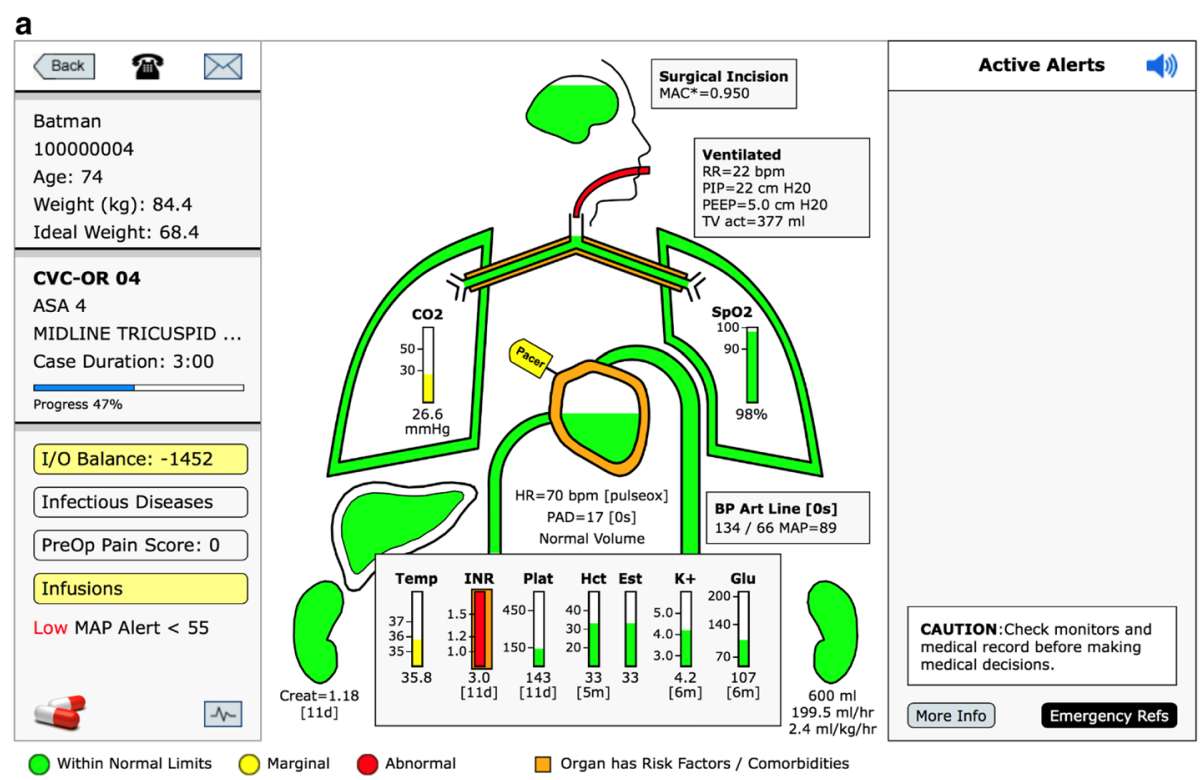

b

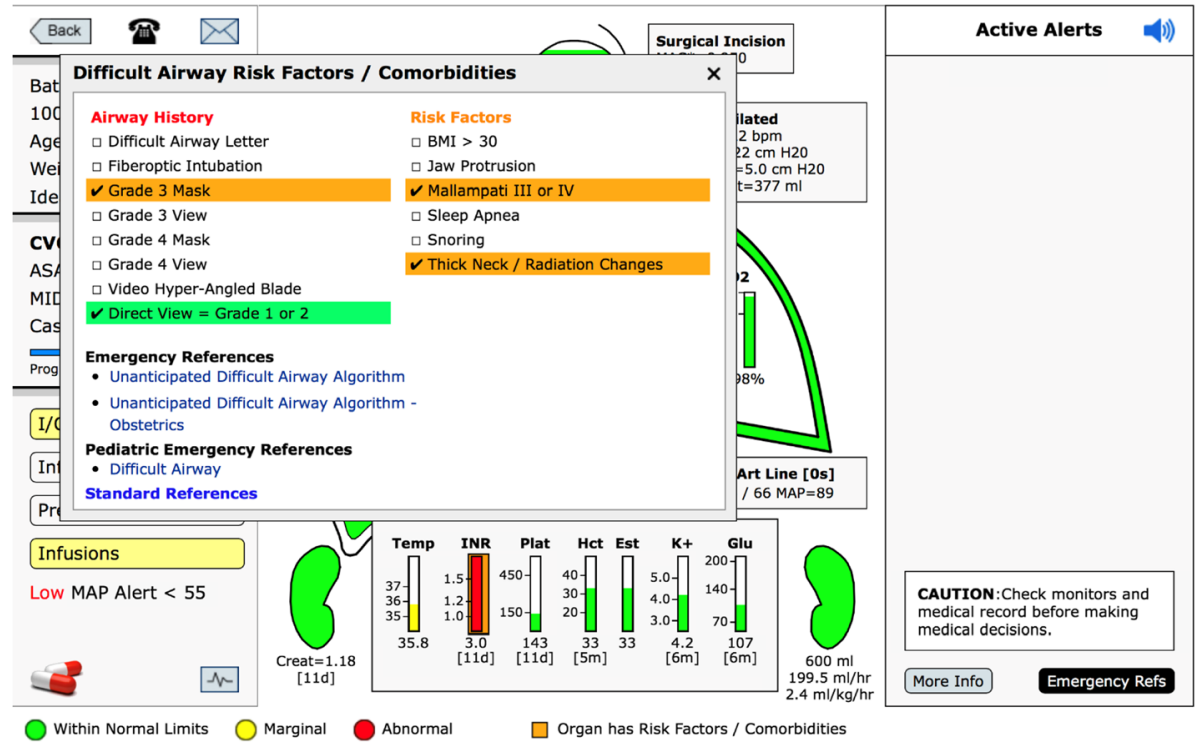

Fig. 6 Endotracheal Tube (ET). a If there is a history of difficult mask ventilation (Grade 3 or 4) or intubation (Grade view 3 or 4 ) the ET tube will be red, as noted above. If there are risk factors for mask ventilation or intubation the ET tube will be orange. If there is a history of easy mask ventilation and intubation the ET tube will be green as noted in Fig. 5. b To see the specific findings, open the ET tube window. At the bottom of this window is a link to the algorithm for unanticipated difficult airway in adults, obstetrics, and pediatrics

icon displays the heart window which lists the Revised Cardiac Risk Indicators (RCRI) and highlights the factors present in this patient, Fig. 8a [29]. It also includes coronary interventions and other cardiac diseases. If the patient has a pacemaker or an implantable cardioverter defibrillator (ICD) there is a small icon attached to the heart, Fig. 7a and Fig. 8b. The window also includes links to adult and pediatric emergency cardiac protocol and references.

The fluid level within the heart is determined by the calculated fluid balance, I\&O. The levels are described in the $\mathrm{I} \& \mathrm{O}$ section.
If there is an invasive method of assessing fluid status such as, systolic pressure variation (SPV), peak pulse pressure variation $(\mathrm{PPV})$, central venous pressure $(\mathrm{CVP})$, or pulmonary artery diastolic pressure (PAD), the fluid level within the heart will be guided by those values [30]. (See color limits in Additional File 1).

In cases where the estimated blood loss (EBL) is greater than $20 \%$ of the EBV and there is no objective measure of cardiac filling as mentioned above, then the area within the heart is white with the text "need data". There is also a text alert in the upper right noting the high level of blood 
a

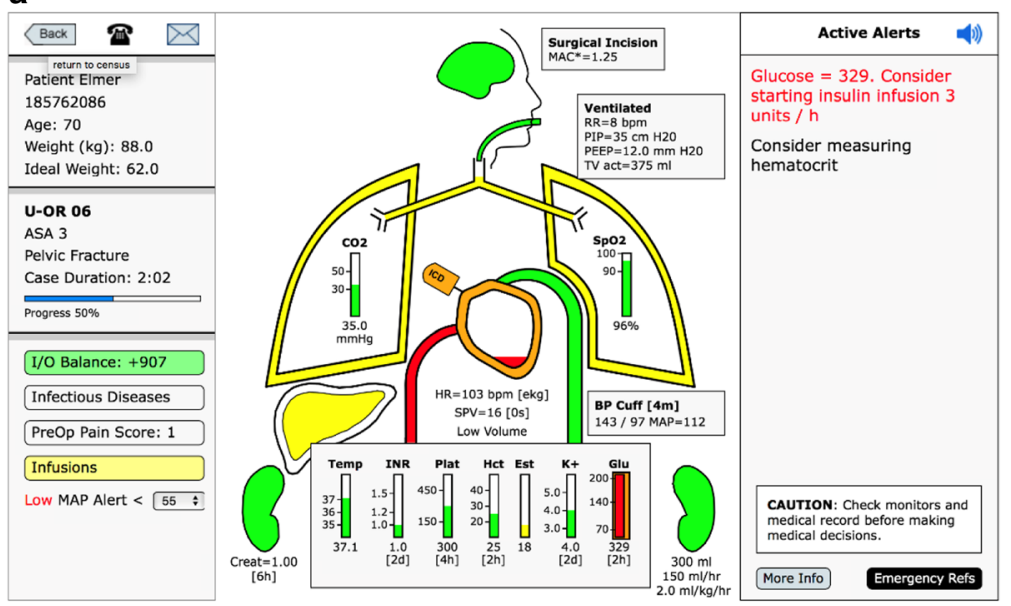

O Within Normal Limits O Marginal Abnormal $\square$ Organ has Risk Factors / Comorbidities

Click the various organ systems, buttons, and bar charts for more information. Not all features are enabled.

b

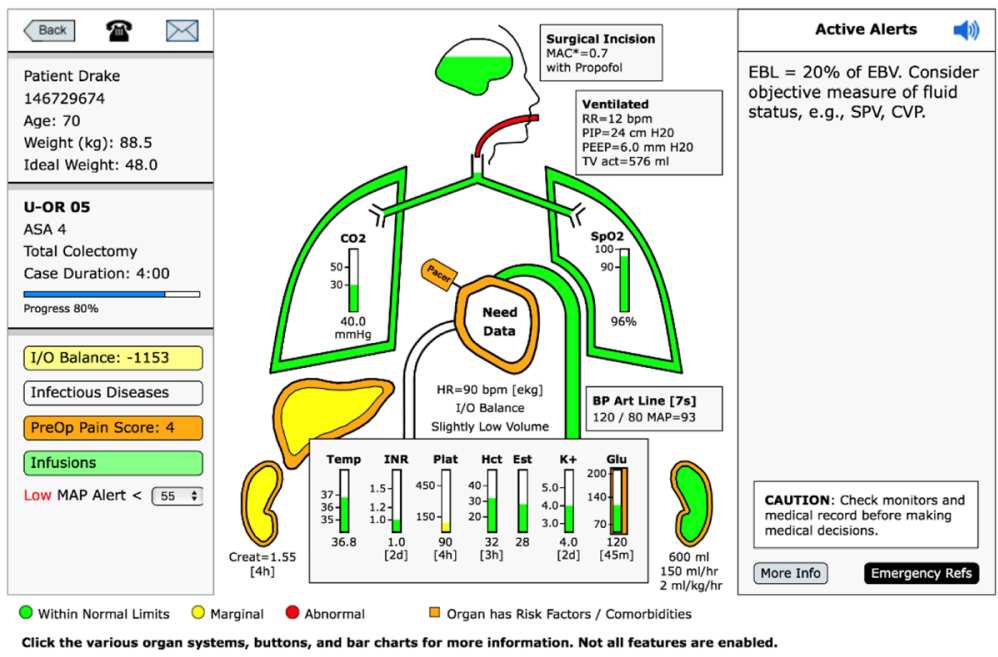

C

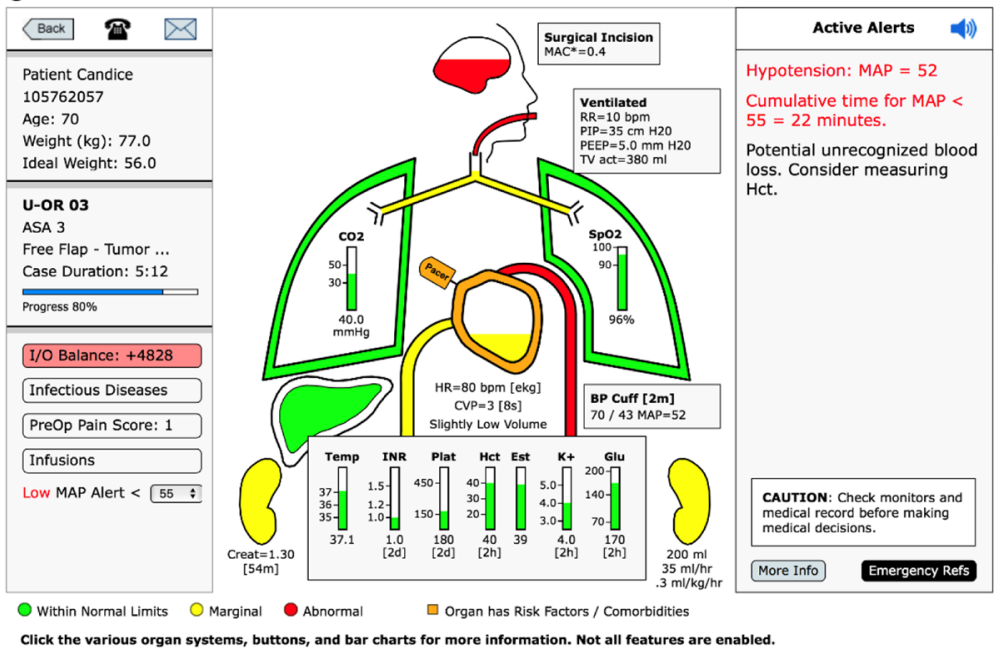

Fig. 7 (See legend on next page.) 
(See figure on previous page.)

Fig. 7 Heart. a The fluid level within the heart is represented as green, yellow, or red depending on the I\&O balance unless there is an invasive measure of fluid status, such as, SPV or CVP. In Fig. 7a note the heart has an ICD (implantable cardiac defibrillator) represented by the small icon attached to the upper left side of the heart. $\mathbf{b}$ If there is no invasive assessment of cardiac filling and the estimated blood loss is $\geq 20 \%$ or the estimated blood volume the inside of the heart will not have a fluid level, but a text alert stating "Need Data." A text alert in the upper right side of the display, stating "Consider Objective Measure of Fluid Status, e.g. SPV, CVP.". c If the I\&O balance is positive ( $\geq 60 \%$ of the EBV) but this coincides with an objective filling measures of SPV, PPV, CVP, or PAD all showing the heart has a very low filling volume and the most recent hematocrit is over $30 \mathrm{~min}$ old, a text alert will be triggered stating "Potential Unrecognized Blood Loss - Consider Measuring Hct"

loss and suggesting the provider consider an invasive method of directly assessing fluid status, "Consider objective measure of fluid status, e.g. SPV, CVP," or stroke volume variation (SVV) if available, Fig. $7 \mathrm{~b}$.

If the EBL is $\geq 40 \%$ of the EBV, the above alert is in red. In the situation where the I\&O balance calculates a very positive, $\geq 60 \%$ of EBV but the objective filling measure (SPV, PPV, CVP or PAD) show a very empty heart and the last hematocrit (Hct) is over $30 \mathrm{~min}$ old an alert is activated: "Potential unrecognized blood loss. Consider measuring Hct", Fig. 7c. There is a more in-depth discussion of all the alerts in the Text Alerts section. Systems may also choose to use hemoglobin (Hgb) instead of Hct as their site default.

On the left side of the heart is the icon of the inferior vena cava. Its color is always the same as the heart because the fluid level represents the filling of the heart.

\section{Blood pressure/aortic arch}

The aortic arch coming out of the right side of the heart signifies the blood pressure (BP). It expands with the contraction of the heart at the heart rate. It is color-coded for BP, in the normal range is green when MAP $\geq 60 \mathrm{mmHg}$, yellow when MAP $<60$ and red when $\mathrm{MAP}<55 \mathrm{mmHg}$. At $\mathrm{MAP}<55 \mathrm{mmHg}$ there is also an audible alert of three tones of decreasing pitch, signifying a very low BP. This also triggers a red scrolling text alert in the upper right of the alerts section. When the cumulative time of MAP $<55 \mathrm{mmHg}$ is $\geq 10 \mathrm{~min}$ there is a permanent red text alert at the top of the Alert Section showing with cumulative time the MAP has been $<55 \mathrm{mmHg}$ for this case, Fig. 8b [7]. The actual BP is displayed along with age of the last BP measurement. When the BP has not been recorded for greater than $5 \mathrm{~min}$ there is alert and $\geq 10 \mathrm{~min}$ the BP measurement turns red.

\section{Liver}

Under the right lung is the liver icon. If it is highlighted in orange, it means there is a history of hepatic disease, which is noted by opening the liver window. If the internal part of the liver is yellow, it means the liver function studies are out of the normal range, also shown in the window.

\section{Kidneys}

On either side of the lab values are the kidneys. The kidney on the left side designates the chronic renal function, green if the creatinine is in the normal range $(<1.2 \mathrm{mg} / \mathrm{dl})$, yellow if it's marginal, creatinine $\geq 1.2 \mathrm{mg} / \mathrm{dl}$ but $<1.5 \mathrm{mg} / \mathrm{dl}$, and red (abnormal) if the creatinine is $\geq 1.5 \mathrm{mg} / \mathrm{dl}$. The kidney on the right of the display shows the current urine output in total $\mathrm{ml} / \mathrm{h}$. and $\mathrm{ml} / \mathrm{kg} / \mathrm{h}$. If the $\mathrm{ml} / \mathrm{kg} / \mathrm{h} .<0.5 \mathrm{mg} / \mathrm{dl}$ the kidney is yellow, Fig. 7c.

\section{Labs}

Body temperature and laboratory values are at the bottom, Fig. 2. These labs all have green, yellow and red ranges which could be noted by selecting the icons at the far bottom of the screen, Additional File 1. The international normalized ratio (INR) and glucose columns maybe highlighted in orange if the patient has medications affecting the bleeding for INR, or if the patient has diabetes, respectively. The system will display a text alert of "No glucose recorded" if the patient is a diabetic.

The Hct is displayed in the center with an estimated (Est) Hct to its right. The Est Hct is derived by equation using most recent Hct, EBV, EBL and blood transfused, see the equation in the window, Fig. 9 [20,31].

\section{Active alerts}

On the right side of the display is the active alert section. There are a total of 54 alerts, Additional File 2. These text alerts come in three levels of severity: black text are informational, red text are important and scrolling red text require an immediate attention. The vast majority of alerts are actionable items which when acted upon as in documenting the AIMS or by lab values retrieved, the alert disappears. There are two exceptions. When the MAP drops $<55 \mathrm{mmHg}$ for more than $10 \mathrm{~min}$ the red alert stays in the upper right and presents the cumulative time the MAP has been below $55 \mathrm{mmHg}$, Fig. 8b [7]. The other alert which remains occur when bolus vasopressors are given. The rationale for having these alerts remain is to allow this information to be clearly noted/identified when there is a transition of care during a hand-over.

All other alerts are impacted by actions that are taken by the provider and will disappear when the appropriate action is taken. For example, if the last glucose measured 


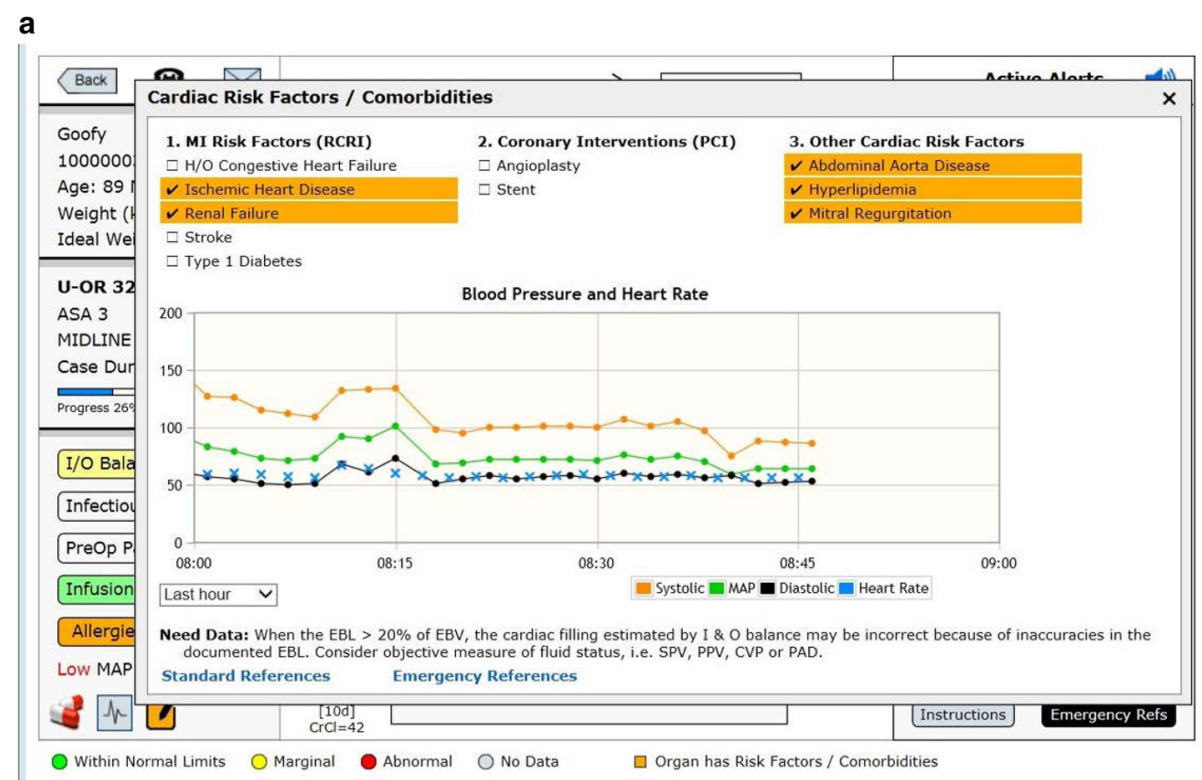

b

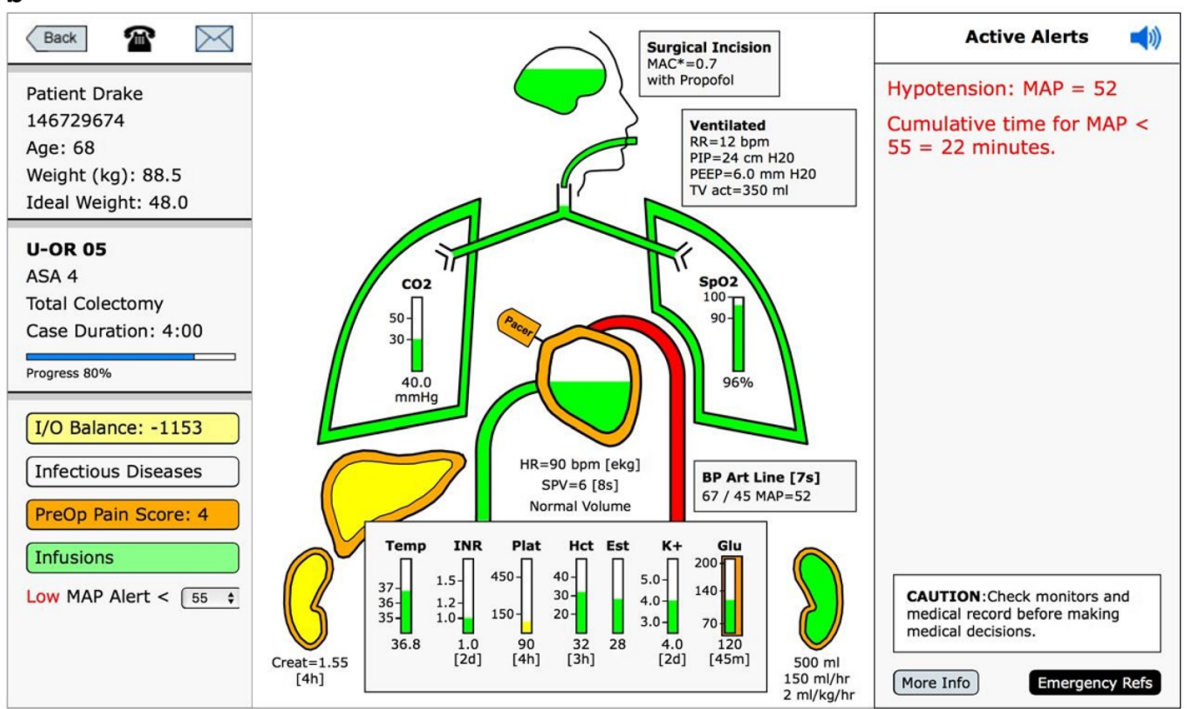

Fig. 8 a Cardiac Risk Factors. Above the patient has cardiac co-morbidities and the heart is outlined in orange. When the heart is touched a cardiac co-morbidity window opens showing risk factors and co-morbidities. Under one are the revised cardiac risk index (RCRI) risk factors. To the left are other coronary interventions and to the left of that are other cardiac risk factors. The risk factors for this patient are highlighted in orange. $\mathbf{b}$. Hypotension Color and Text Alert. Note above the aortic arch is red signifying the mean arterial pressure is $<55 \mathrm{mmHg}$. In the upper right side of the display a text alert signifies the blood pressure as well as the number of accumulated minutes the mean arterial pressure has been $<55 \mathrm{mmHg}$

in a patient record is over $200 \mathrm{mg} / \mathrm{dl}$ there will be an alert suggesting that a repeat glucose be measured. Once it has been measured if it is $<200 \mathrm{mg} / \mathrm{dl}$ the alert will disappear if it's $\geq 200 \mathrm{mg} / \mathrm{dl}$ and the patient is not an outpatient and the case is longer than $1 \mathrm{~h}$ an alert will appear suggesting treatment with insulin [16]. Once there is documentation of insulin therapy that alert will disappear and a 60 min clock will start. At $60 \mathrm{~min}$, if there is no repeat glucose lab noted an alert will appear recommending a repeat glucose measurement. This alert logic has recently been documented to improve glucose management [16]. Another example is if the TV is calculated to be $\geq 10 \mathrm{ml} / \mathrm{kg}$ ideal body weight there will be an alert displayed which suggests the appropriate TV to be within 6 to $8 \mathrm{ml} / \mathrm{kg}$, Fig. 5 [8].

There are a series of alerts with respect to transfusion therapy suggesting when to measure labs/ coagulation studies, when to consider treatment with blood and blood products and calcium. These follow standard algorithms for transfusion therapy [32]. 


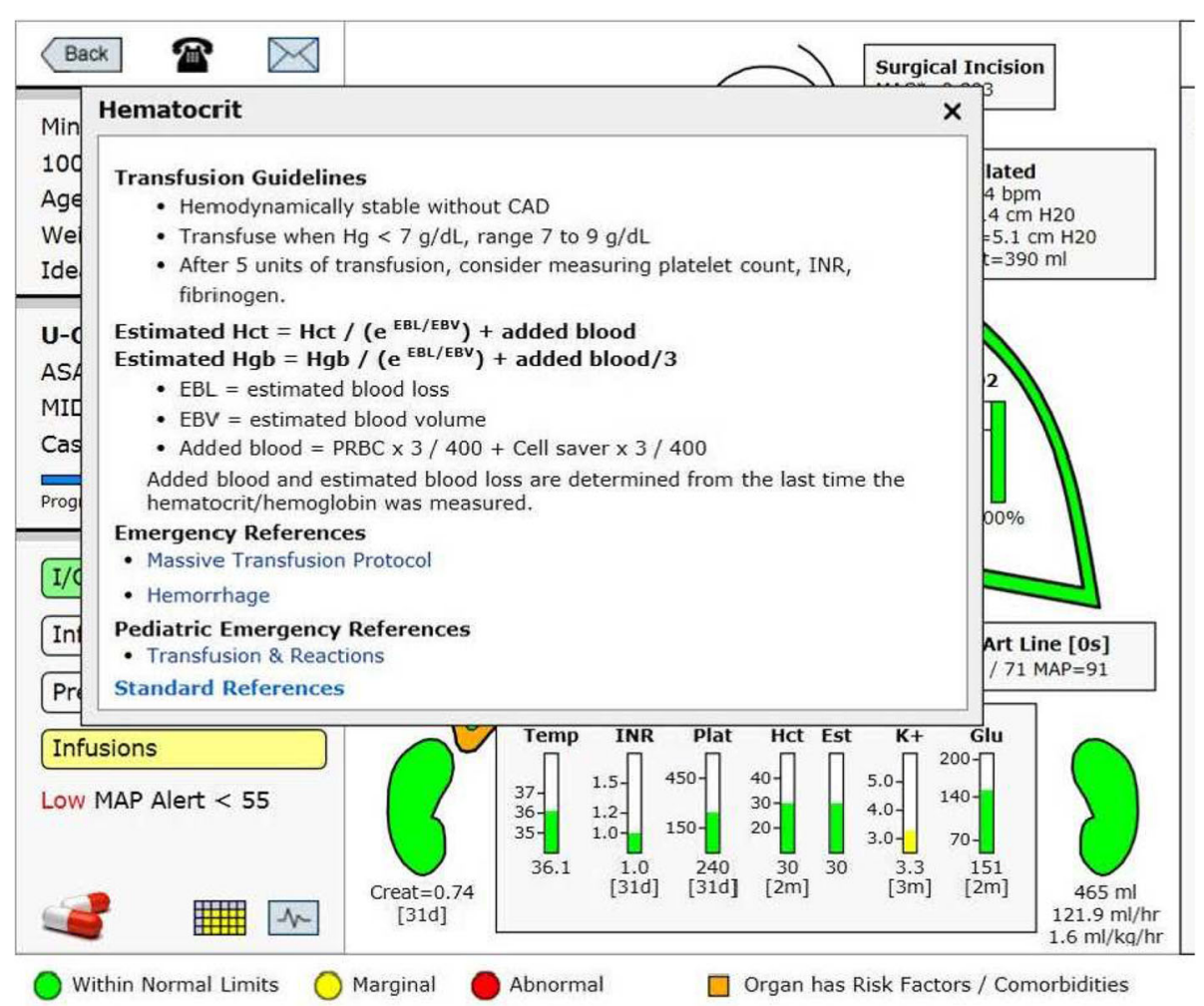

Fig. 9 Estimated Hematocrit. This window opens when the estimated hematocrit or hematocrit columns are touched. It presents the equation used to determine an estimated hematocrit based on the last hematocrit drawn, the estimated blood loss, any blood given to the patient and the estimated blood volume, which is based on height, weight and gender. As soon as a new hematocrit is drawn and retrieved by the system the estimated hematocrit and hematocrit will be equal until there is a change in either blood loss or blood given to the patient

\section{Anesthesiology performance improvement and reporting exchange (ASPIRE) metrics}

ASPIRE is a quality collaborative supported by Blue Cross Blue Shield of Michigan developed and coordinated by the University of Michigan. It is a collaborative of 30 hospital representatives of anesthesia departments in Michigan who jointly have determined a series of quality of care metrics [33]. AlertWatch has coordinated its alerts with these ASPIRE quality metrics.

\section{Emergency references}

Finally, in the lower right-hand corner of the screen is an emergency reference tab. This links to the adult emergency reference algorithms for the most common emergent events in the operating room [27].

\section{Configurable alerts and limits}

The alerts and the limits on the alerts are all configurable upon installation at any institution. The FDA clearance process required the default alerts in the system have evidence in the literature and/or documented expert opinion in case the institution chooses to proceed with the default alerts and alert limits programed in AlertWatch OR.

\section{Discussion}

The aviation industry is several decades ahead of medicine in taking advantage of large amounts of real-time data, to develop algorithms, protocols, and integrating them in a manner that increases situational awareness in order to improve safety and quality. This information is displayed in a readily identifiable format to enable the pilots to interpret and utilize these data while flying the aircraft: the primary flight display within the glass cockpit turns data into actionable information.

The explosion of medical data ranging from the EMR to the human genome, will provide the opportunity to develop more high resolution guidelines and protocols down to an individual patient level with their known comorbidities undergoing a specific procedure that can change on a minute to minute basis based on additional live data being received. To take advantage of this opportunity, there must be a way the care giver at the bedside can navigate these personalized recommendations and be alerted to these guidelines at the time, place and patient to which they should be applied. The increasing presence of monitors in critical care units has found that increasing the number of audible alerts to an array of ana$\log$ waveforms with only simplistic high/low threshold 
alarms has frequently resulted in alarm fatigue rather than materially enhancing the care and safety of the patient [34].

One of the principles in developing AlertWatch OR was to provide an integrated display with a hierarchy of alerts presented in multiple, and some would say in a redundant manner; without producing alarm fatigue. This has been approached by presenting information in a hierarchy of color displays: red, yellow, green and orange; text alerts, black text, red text and scrolling red text; and some audible and/or paging alerts associated with the highest risk issues, e.g. severe hypotension, severe hypoglycemia.

Table 1 presents the number of alerts and categories of alerts for a seven-day period at the University of Michigan. As noted in this table, there were 5970 alerts in 1755 cases with an average case length of $126 \mathrm{~min}$. Of those alerts, 1568 were for documentation and billing reminders, leaving 4402 alerts relating to all others aspects of the display. Drug dosing, antibiotic timing and train-of-four alerts accounted for 668 leaving 3734 more physiologic alerts. This oneweek sample found approximately 2.5 text alerts per case, or at our institution 1.5 clinical text alerts per hour, Table 1. The most common of which was significant hypotension for greater than $10 \mathrm{~min}$. Over this one-week period there were 314 red scrolling

Table 1 Text Alerts During One Week of Cases at University of Michigan ORs: 1755 cases

\begin{tabular}{|c|c|c|c|}
\hline & Alerts & Alerts/Case & $\begin{array}{l}\text { Alerts/Hour of } \\
\text { Anesthesia }\end{array}$ \\
\hline Total & 5970 & 3.4 & 1.7 \\
\hline Documentation/Billing & 1568 & 0.9 & 0.45 \\
\hline Clinical Alerts: & 4402 & 2.5 & 1.25 \\
\hline $\begin{array}{l}\text { Drugs/Antibiotics/Neuromuscular } \\
\text { Blocker/ Train-of-Four }\end{array}$ & 668 & 0.38 & 0.8 \\
\hline Glucose High/Low & 118 & 0.07 & \\
\hline Cardiac & 470 & 0.26 & 0.13 \\
\hline Blood Pressure & 2466 & 1.4 & 0.7 \\
\hline Ventilation & 589 & 0.3 & 0.15 \\
\hline $\begin{array}{l}\text { Red Scrolling Text/Beeping } \\
\text { Tone/Paging: }\end{array}$ & 314 & 0.2 & 0.01 \\
\hline Glucose $<60 \mathrm{ml} / \mathrm{dl}$ & 2 & 0.18 & \\
\hline $\mathrm{HCT}<18 \%$ & 9 & 0.01 & \\
\hline $\mathrm{MAP}<55 \mathrm{mmHg}(>10 \mathrm{~min})$ & 303 & 0.17 & 0.08 \\
\hline
\end{tabular}

Legend: Above are the total number of text alerts triggered by AlertWatch OR during seven days at the University of Michigan operating rooms. The total alerts at the top are 5970 alerts were triggered in 1755 cases. They are subdivided into documentation and billing alerts (the largest number, 1568) drug alerts, and physiologic alerts. At the bottom, the highest risk alerts, which are associated with scrolling red text and a beep tone or a page, are for low glucose, low hematocrit or mean blood pressure below $55 \mathrm{mmHg}$ for more than $10 \mathrm{~min}$. The most common of these serious alerts was for hypotension. AlertWatch ${ }^{\circledast}$ OR stores no clinical data. It only stores alerts for quality assurance purposes which are not part of the medical record alerts associated with a page and/or a beep tone (2 glucose $<60 \mathrm{mg} / \mathrm{dl}, 9$ hematocrit $<18 \%$, and 303 mean blood pressure $<55 \mathrm{mmHg}$ for $10 \mathrm{~min}$ ) in approximately once every five cases.

\section{Conclusion}

AlertWatch ${ }^{\ominus}$ was developed over a five-year period at the University of Michigan. It was inspired by the aviation industry's development of the primary flight display/multifunction display used in the glass cockpit display of modern aircraft. The aviation industry has led the way in safety. Although AlertWatch OR tries to emulate what has been found to be extremely useful in aviation, the physics of flying a plane are likely less variable than the physiology of managing a complex patient in the operating room. This is one reason why the AlertWatch OR icon display is more complex than the horizon display on a plane's flight monitor, shown in Fig. 1. Clearly there is much more variability patient-topatient and case-to-case and many of the "rules" are only as good as the most recent literature and guidelines on which those rules are based. The development of AlertWatch $^{\circ}$ is our attempt at emulating aviation's processes to increase situational awareness with the goal of achieving their extremely high levels of safety.

\section{Availability and requirements}

Project Name: AlertWatch OR.

Project Home Page: http://www.alertwatch.com

Operating System: Platform independent.

Programming Language: SQL/P-SQL, C\#.NET with Java script front-end.

Other Requirements: Java enabled web browser.

License: No license required for viewing demo. FDA cleared software medical device requires licensing for installation.

Any restrictions to use by non-academics: No

\section{Additional files}

Additional file 1: List of Color Limits. These color limits have received FDA Clearance, and they also can be changed at the institution level. (PDF $96 \mathrm{~kb}$ )

Additional file 2: List of Text Alerts. There are a total of 54 alerts. These text alerts come in three levels of severity: black text are informational, red text are important and scrolling red text require an immediate attention. (PDF $66 \mathrm{~kb}$ )

\section{Abbreviations}

AIMS: Anesthesia Information Management System; ASA: American Society of Anesthesiologists; BIS: Bispectral index; BP: Blood pressure; COPD: Chronic obstructive pulmonary disease; CVP: Central venous pressure;

EKG: Electrocardiogram; EMR: Electronic medical record; Est: Estimated; FDA: Food and Drug Administration; Hct: Hematocrit; Hgb: Hemoglobin; I\&O: Intake and output; ICD: Implantable cardioverter defibrillator; ICD-10: International classification of diseases; INR: International normalized ratio; IV: Intravenous; MAC*: Minimum alveolar concentration; MAP: Mean 
arterial pressure; NPO: Nothing by mouth; OR: Operating room; PACU: Post Anesthesia Care Unit; PAD: Pulmonary artery diastolic pressure; PPV: Peak pulse pressure variation; SPV: Systolic pressure variation; SW: Stroke volume variation; TIVA: Total intravenous anesthesia

\section{Acknowledgements}

Not Applicable.

\section{Funding}

Not Applicable.

\section{Availability of data and materials}

This is proprietary software for this FDA cleared medical device. We are unable to provide the software, which is in excess of 10,000 lines of code. There is no repository as this software does not store data.

\section{Authors' contributions}

$K T$ was a major contributor in designing the application and writing the manuscript; JM is a research assistant who has helped with writing the manuscript, researching references and organization of the project; JG was the first programmer to initiate the design of the application with its alerts. $\mathrm{He}$ is also the primary programmer for the interface and the algorithm implementation; TT is a programmer, primarily involved in database development to drive the application; JA has assisted in the design and development to drive the application; and JB helped in the design and modification of the application and its presentation. All authors on this manuscript have been involved in some aspect of the design and development of AlertWatch ${ }^{\circledast}$ OR. All authors read and approved this manuscript.

\section{Ethics approval and consent to participate Not Applicable.}

\section{Consent for publication.}

Not Applicable

\section{Competing interests}

$\mathrm{KT}$ is the founder and president of AlertWatch and hold patents and equity interest in the company; JM is a founder and research assistant and holds an equity interest in the company; JG is a founder and chief technology officer and hold an equity interest in the company; $\Pi$ is a founder and a clinical research analyst and holds an equity interest in the company; JA is the chief executive officer and holds equity interest in the company; JB is a founder and vice president of patient safety and holds an equity interest in the company.

\section{Publisher's Note}

Springer Nature remains neutral with regard to jurisdictional claims in published maps and institutional affiliations.

\section{Author details}

${ }^{1}$ Department of Anesthesiology, University of Michigan Medicine, $1500 \mathrm{E}$. Medical Center Drive, Ann Arbor, MI 48109, USA. ${ }^{2}$ AlertWatch Headquarters, 330 E. Liberty Street, Ann Arbor, Ml 48104, USA.

Received: 21 June 2017 Accepted: 19 January 2018 Published online: 05 February 2018

\section{References}

1. Statistical Summary of Commercial Jet Airplane Accidents. Worldwide Operations: 1959-2015. www.boeing.com/news/techissues/pdf/statsum.pdf. Accessed 31 Aug 2016

2. Glass Cockpit. NASA Fact Sheet. http://www.nasa.gov/centers/langley/news/ factsheets/Glasscockpit.html. Accessed 26 Aug 2016.

3. American Society of Anesthesiologists Standards for Basic Anesthetic Monitoring. (Committee of Origin: Standard and Practice Parameters.) Approved by the ASA House of Delegates on October 21, 1986. [Online] http://www.asahq.org/qualityand-practice-management/standards-guidelines-and-related-resources/standardsfor-basic-anesthetic-monitoring. Accessed 25 Aug 2016.
4. Neily J, Mills PD, Young-Xu Y, et al. Association between implementation of a medical team training program and surgical mortality. JAMA. 2010;304(15): 1693-700.

5. Haynes $A B$, Weiser TG, Berry WR, et al. A surgical safety checklist to reduce morbidity and mortality in a global population. N Engl J Med. 2009;360: 491-9.

6. Kheterpal S, O'Reilly M, Englesbe MJ, et al. Preoperative and intraoperative predictors of cardiac adverse events after general, vascular, and urological surgery. Anesthesiology. 2009;110:58-66.

7. Walsh M, Devereaux PJ, Garg AX, Kurz A, Turan A, Rodseth RN, Cywinski J, Thabane L, Sessler DI. Relationship between Intraoperative mean arterial pressure and clinical outcomes after noncardiac surgery. Anesthesiology. 2013;119:507-15.

8. Futier E, Constantin JM, Paugam-Burtz C, et al. A trial of intraoperative low-tidal volume ventilation in abdominal surgery. N Engl J Med. 2013; 369:428-37.

9. Monk TG, Bronsert MR, Henderson WG, Mangione MP, STJ S-P, Bentt DR Nguyen JD, Richman JS, Meguid RA, Hammermeister KE. Association between Intraoperative hypotension and hypertension and 30-day postoperative mortality in noncardiac surgery. Anesthesiology. 2015;123:307-19.

10. Sun LY, Wijeysundera DN, Tait GA, Beattie WS. Association of Intraoperative Hypotension with acute kidney injury after elective noncardiac surgery. Anesthesiology. 2015;123:515-23.

11. van Waes, JA et al Association between Intraoperative hypotension and myocardial injury after vascular surgery Anesthesiology 2016, V 124, No 1 p 35-44.

12. Kotagal M, Symons RG, Hirsch IB, Umpierrez GE, Dellinger EP, Farrokhi ET, Flum DR, (CHASE Alliance). Perioperative hyperglycemia and risk of adverse events among patients with and without diabetes. Ann Surg 2015; 261(1):97-103.

13. Chappell D, Jacob M, Hofmann-Kiefer K, et al. A rational approach to perioperative fluid management. Anesthesiology. 2008;109:723-40.

14. Kheterpal S, Shanks A, Tremper KK. Impact of a Novel Multiparameter Decision Support System on Intraoperative Processes of Care and Postoperative Outcomes. Anesthesiology 2018; 128:272-82.

15. Tremper KK. Anesthesiology: from patient safety to population outcomes. The $49^{\text {th }}$ annual Rovenstine lecture. Anesthesiology. 2011;114:755-70.

16. Sathishkumar S, Lai M, Picton P, Kheterpal S, Morris M, Shanks A, Ramachandran SK. Behavioral modification of Intraoperative hyperglycemia management with a novel real-time audiovisual monitor. Anesthesiology. 2015;123:29-37.

17. AlertWatch ${ }^{\oplus:}$ OR, FDA 510(k) Clearance. (Accessed on 29 Aug. 2016, at https://www.accessdata.fda.gov/cdrh_docs/pdf13/K130401.pdf).

18. Asada, K. Chromatic vision simulator; 2013, http://asada.tukusi.ne.jp/ cvsimulator/e/. Accessed 18 Oct 2017.

19. Robinson JD, Lupkiewicz SM, Palenik L, Lopez LM, Ariet M. Determination of ideal body weight for drug dosage calculations. Am J Health Syst Pharm. 1983;40(6):1016-9.

20. Nadler S, Hidalgo J, Bloch T. Prediction of blood volume in normal human adults. Surgery. 1962;51:224-32.

21. Cortes DO, Barros TG, Njimi H, Vincent JL. Crystalloids versus colloids: exploring differences in fluid requirements by systematic review and meta-regression. Anesth Analg. 2015;120:389-402.

22. Kaye AD. Fluid Management, Chapter 23. In: Miller RD, Pardo Jr MC, editors. Basics of anesthesia. 6th ed. Philadelphia, PA: Elsevier Saunders; 2011. p. 364-71.

23. Mashour GA, Esaki RK, Vendervest JC, Shanks A, Kheterpal S. A novel electronic algorithm for detecting potentially insufficient anesthesia: implications for the prevention of intraoperative awareness. J Clin Monit Comput. 2009;23:273-7.

24. Mashour GA, Shanks A, Tremper KK, Kheterpal S, Turner CR. Prevention of intraoperative awareness with explicit recall in an unselected surgical population. Anesthesiology. 2012;117:717-25.

25. Kheterpal S, Han R, Tremper KK, Shanks A, Tait AR, O'Reilly M, Ludwig T. Incidence and predictors of difficult and impossible mask ventilation. Anesthesiology. 2006;105:885-91.

26. Kheterpal S, Healy D, Aziz M, et al. Incidence, predictors and outcome of difficult mask ventilation combined with difficult Laryngoscopy. Anesthesiology 2013; 119:1360-1369.

27. Arriaga AF, Bader AM, Wong JM, et al. Simulation-based trial of surgical-crisis checklists. N Engl J Med. 2013;368:246-53. 
28. Pediatric Difficult Airway Protocol in Pedicrisis Critical Event Cards, Society for Pediatric Anesthesia, revised November 19, 2014.

29. Lee TH, Marcantonio ER, Mangione CM, et al. Derivation and prospective validation of a simple index for prediction of cardiac risk of major noncardiac surgery. Circulation. 1999;100:1043-9.

30. Mathis MR, Schechtman SA, Engoren MC, Shanks AM, Thompson A, Kheterpal S, Tremper KK. Arterial pressure variation in elective noncardiac surgery: identifying reference distributions and modifying factors. Anesthesiology. December 2016; online: http://anesthesiology.pubs.asahq. org/article.aspx?articleid=2592737\& resultClick=3.

31. Feldman JM, Roth JV, Bjoraker DG. Maximum blood savings by acute Normovolemic Hemodilution. Anesth Analg. 1995;80:108-13.

32. Adult Blood Transfusion Clinical Guidelines, University of Michigan Hospitals and Health Centers. Approved by the UMHHC Executive Committee for Clinical Affairs on March 24, 2009.

33. Anesthesiology performance improvement and reporting exchange (ASPIRE). Current Performance Measures. https://mpog.org/quality/ourmeasures/. Accessed 20 Sept 2016.

34. Bonafide CP, Lin R, Zander M, Graham CS, Paine CW, Rock W, Rich A, Roberts KE, Fortino M, Nadkarni VM, Localio AR, Keren R. Association between exposure to nonactionable physiologic monitor alarms and response time in a children's hospital. J Hosp Med. 2015;10:345-51.

\section{Submit your next manuscript to BioMed Central and we will help you at every step:}

- We accept pre-submission inquiries

- Our selector tool helps you to find the most relevant journal

- We provide round the clock customer support

- Convenient online submission

- Thorough peer review

- Inclusion in PubMed and all major indexing services

- Maximum visibility for your research

Submit your manuscript at www.biomedcentral.com/submit 